\title{
PERCEPÇÃO DE IDOSOS COM DOENÇAS CRÔNICAS SOBRE A PERDA DA FUNCIONALIDADE E DA AUTONOMIA
}

\author{
Maria Vieira de Lima Saintrain', Caroline Barbosa Lourenço', Caroline Ferreira Martins', Ana \\ Nery Melo Cavalcante', Rosa Lívia Freitas de Almeida' e Raimunda Magalhães da Silva' \\ 1 Universidade de Fortaleza, Fortaleza-Ceará-Brasil.mvlsaintrain@yahoo.com.br_kerol25@yahoo.com.br \\ carolfm.lessa@gmail.com;ananery.melo@bol.com.br;rliviafa@unifor.br_;rmsilva@unifor.br
}

\begin{abstract}
Resumo. Introdução:O envelhecer natural e saudável pode ser objeto de mudanças estruturais e funcionais, manifestando-se como natural do ser humano. Objetivo: Conhecer a percepção dos idosos sobre a perda da funcionalidade e da autonomia causada pelas doenças crônicas. Método: Pesquisa qualitativa desenvolvida com 14 idosos em área urbana, adstrita à Unidade Básica de Saúde, em município do Nordeste brasileiro. Os idosos responderam entrevista semiestruturada, utilizando perguntas abertas para permitir o fluxo livre de suas respostas. Estas foram transcritas na íntegra e, para tratamento dos dados, optou-se pela Análise de Conteúdo preconizada por Bardin. A recolha dos indicadores assentou-se nas indagações: Quais as interferências das doenças crônicas nas condições de vida dos idosos? Como os idosos se sentem com a perda da funcionalidade? De que maneira eles se sentem com a sua autonomia?. Resultado: Ao categorizar as falas, surgem sentimentos de tristeza, vontade de morrer, aflições quanto ao modo de viver na familia e na sociedade. A perda da funcionalidade dimensiona constrangimento consigo mesmo, enquanto a autonomia reflete a percepção sobre o prejuízo da habilidade de cuidar de si, a falta de interação com os outros e seu papel social.Conclusão:O idoso com vulnerabilidade funcional e de autonomia denota sentimentos que afetam a compreensãoda liberdade, a conduta do cuidar de si e os valores na tomada de decisão.
\end{abstract}

Palavras-chave: Doenças Crônicas; Funcionalidade; Autonomia; Pessoa Idosa.

\section{CHRONICALLY ILL OLDER ADULTS' PERCEPTION OF LOSS OF FUNCTIONALITY AND AUTONOMY}

\begin{abstract}
Introduction: Natural and healthy aging can undergo structural and functional changes and manifest itself as a natural human process. Objective: To know older adults' perception of loss of functionality and autonomy caused by chronic diseases. Method: This is a qualitative study of 14 older people in an urban area covered by a Primary Health Care center in a municipality in Northeastern Brazil. Semi-structured interviews were held with the participants using open-ended questions to allow the free flow of their answers. They were transcribed verbatim and data were treated using Bardin's Content Analysis. Data collection was based on the questions: what are the interferences of chronic diseases in older adults' living conditions? How do older adults feel about loss of functionality? and How do they fell about their autonomy? Results: The categorization of the responses revealed feelings of sadness, desire to die, and anguish related to the mode of life within the family and society. Loss of functionality caused embarrassment about themselves and autonomy reflected their perception of self-care, lackof interaction with others and their social role. Conclusion: Older adults with impaired functionality and autonomy experience feelings that affect their understanding of freedom andpractice of self-care and decision-making skills.
\end{abstract}

Keywords: Chronic Diseases; Functionality; Autonomy;Perception; Older Adult.

\section{INTRODUÇÃO}

No Brasil, a Política Nacional de Saúde do Idoso assim considera pessoas com 60 anos ou mais, assegurando, explicitamente, os direitos primordiais à saúde dessa população (Brasil, 2006). 
Nesta conjuntura, o rápido aumento da população idosa e da expectativa de vida, resultante, sobretudo, da melhoria na qualidade dos serviços de saúde e das condições de vida, acarretou mudanças epidemiológicas irreversíveis, caracterizadas por doenças crônicas e múltiplas que perduram por anos (Brasil, 2017; Pereira, Souza \& Vale, 2015).

Se, entretanto, esses anos adicionais forem reprimidos por declínios na capacidade funcional, as implicações para os idosos e para a sociedade podem ser muito negativas (WHO, 2015).

O envelhecer natural e saudável pode experimentar uma série de mudanças estruturais e funcionais, que são cumulativas, progressivas, intrínsecas e deletérias, as quais se manifestam como algo natural do ser humano (Sgarbieri\& Pacheco, 2017).

Assim sendo, o envelhecimento é, evidentemente, inevitável, marcado por uma perda progressiva de funções sensoriais e motoras, aumentando, consequentemente, a vulnerabilidade às doenças crônicas. Estes agravos podem afetar a funcionalidade, a mobilidade e a independência, impossibilitando a pessoa de desenvolver um envelhecimento saudável e autônomo (Leandro-Franco \&Murta, 2014).

Com vistas a oferecer meios para a reorganização dos serviços de saúde, o Ministério da Saúde do Brasil lança a Estratégia para promoção do envelhecimento saudável, cuja meta configura a "linha de cuidado para o atendimento à pessoa idosa", como primazia no nível de independência e autonomia para atividades cotidianas, na premissa de reduzir a perda da autonomia, aumentar o desempenho cognitivo e a sobrevida nessa faixa etária (MS/Brasil, 2017).

Malgrado os avanços relativos ao envelhecimento, ainda se impõe necessário fornecer elementos para o desenvolvimento de ações públicas adequadas a essa população. Dentre os progressos técnicos, científicos e tecnológicos para o envelhecimento, questiona-se: Quais as interferência das doenças crônicas nas condições de vidados idosos? Como os idosos se sentem com a perda da funcionalidade? De que maneira se acham com a sua autonomia?

Com o pressuposto de que as doenças crônicas comprometem as condições fisicas, sociais e comportamentais do idoso, este ensaio tem com objetivo conhecer a percepção dos 
idosos sobre a condição de vida, perda da funcionalidade e da autonomia, causada pelas doenças crônicas.

Como importante passo em direção ao que preconiza a estratégia lançada pelo Ministério da Saúde, a pesquisa ora sob relato buscou informações que possam contribuir no fortalecimento de ações direcionadas à atenção das pessoas idosas mais vulneráveis, segundo os princípios da equidade e da integralidade da saúde.

\section{METODOLOGIA}

Este estudo é de ordem qualitativa, com uma abordagem compreensiva das experiências de vida, envolvendo os aspectos das relações, sentidos, valores, crenças, comportamentos e sentimentos dos idosos (Minayo, 2014). Em demandas qualitativas, os entrevistados são convidados a contar suas histórias, garantido um ritual de respeito, acolhimento e segurança. A interação e a negociação humana são vistas como a base para a criação e entendimento da vida social e de conhecimento (Edwards \& Holland, 2013).

A pesquisa foi desenvolvida com 14 idosos de ambos os sexos, com idade de 60 anos ou mais que residiam em uma área urbana, adstrita à Unidade Básica de Saúde localizada em um município do Estado do Ceará, no Nordeste brasileiro. Os participantes tinham caracteristicas semelhantes em relação às condições sociais, econômicas, educacionais e culturais. Estes foram identificados nos serviços de saúde e após a aplicação de um questionário - de uma pesquisa anterior - para 165 idosos com 60 anos (media de $67.0 \pm$ 6.0 anos), com a finalidade de investigar as atividades da vida diária AVD e atividades instrumentais da vida diária - AIVD.

As atividades inerentes ao instrumento AVD dizem respeito às configurações do autocuidado, como tomar banho, vestir-se, fazer higiene pessoal, transferência, controle de esfíncteres, alimentação. Sua taxionomia é procedida de acordo com a pontuação do somatório de resposta "SIM". Tem como escores um ponto para cada resposta "sim", e classifica-os como Independência, quando o resultado é de seis pontos - Défice Moderado, com quatro pontos, e Défice Severo, com dois pontos (Katz, S.et al., 1983).

Quanto ao instrumento AIVD, estão incluídas oito variáveis referentes às habilidades de mobilidade (usar o telefone, ir a locais distantes utilizando algum meio de transporte, fazer compras, arrumar a casa, lavar roupa, preparar a própria refeição, tomar medicamentos e 
cuidar do dinheiro). Para cada atividade, são propostas três opções: Sem Ajuda (três pontos), com Ajuda Parcial (dois pontos), Não Consegue (um ponto) - tendo como escore máximo 27 pontos. O escore tem significado individual e o declínio à extensão do tempo revela deterioração. O somatório com valores de 19 a 27 pontos classifica-o com independência; de dez a 18 pontos, tem capacidade com assistência e de um a nove pontos tem dependência (Lawton and Brody,1969).

Com os resultados destes instrumentos, identificou-se a necessidade de aprofundar o estudo em relação às doenças crônicas e comprometimento na capacidade funcional e autonomia pessoal. Os 14 idosos acometidos de doenças crônicas autorreferidas (hipertensão arterial, Diabetes Mellitus, reumatismo) foram convidados pessoalmente a participar da entrevista e todos aceitaram, assinando o Termo de Consentimento. A inclusão dos idosos baseou-se nas boas condições físicas e cognitivas para responder a entrevista, havendo-se excluído aqueles com sinais de demência ou com dificuldade de exercer a oralidade.

Realizou-se a coleta de dados no periodo 10 de janeiro a 05 de fevereiro de 2020, por meio de entrevista semiestruturada, aplicada no domicílio por uma das pesquisadoras com experiência na atenção primária de saúde e no cuidado com idoso. A entrevista constou de perguntas abertas a respeito das interferência das doenças crônicas na vida, os sentimentos e percepções quanto a capacidade fisica e autonomia ante a situação de saúde vivenciada.

Os idosos aceitaram gravar a entrevista, a qual se efetivou individualmente em um local adequado depois da visita domiciliar do agente de saúde, com duração de aproximadamente uma hora, acrescida da observação direta do pesquisador. Para garantir o anonimato, os participantes foram classificados pela letra $E$ de entrevista, acompanhada da sequência númerica.

As respostas foram transcritas na íntegra e organizadas de acordo com a Análise de Conteúdo, de Bardin(2018), que preconiza a elaboração sistemática de uma pré-análise do material, exploração deste, codificação, categorização, inferência e interpretação dos dados.

Dentre as técnicas utilizadas para a realização da Análise de Conteúdo, optou-se pelo exame categorial. Esse tipo de análise cuida do desmembramento do discurso em categorias, em que os critérios de escolha e delimitação se orientam pela dimensão 
investigativa dos temas relacionados ao objeto de pesquisa, identificados nos discursos dos pesquisados (Bardin, 2018).

O projeto teve aprovação do Comitê de Ética em Pesquisa da Universidade de Fortaleza, Parecer número 2.726.401/18. Todos os participantes receberam esclarecimento e informações a respeito do trabalho, tendo garantidos o anonimato e a liberdade de retirar-se do estudo a qualquer momento, enquanto aqueles que participaram assinaram o Termo de Consentimento Livre e Esclarecido (TCLE).

\section{RESULTADOS E DISCUSSÃO}

Dos 14 participantes, nove eram mulheres e cinco homens, com idade que variou de 64 a 89 anos. Todos pertenciam à classe socioeconômica de baixa renda, sendo que eram aposentados e recebiam de meio até dois salários-mínimos.

De posse dos achados, foram identificados para a análise os temas sobre a) Interferência das doenças crônicas nas condições de vida; b) Doenças crônicas e o desempenho funcional; c) Percepção da autonomia e a influência na dinâmica da vida.

Vários fatores podem estar relacionados a dependência ou limitação funcional das pessoas idosas, podendo-se destacar fatores sociodemográficos e condição de saúde geral, todos associados ao apoio familiar. Ainda pouco se sabe, entretanto, sobre e a magnitude do influxo da dependência na manutenção da saúde em idosos (Saintrain \& Vieira, 2018).

Estudo realizado por Virtuoso-Júnior, Martins, Roza, Paulo, Ribeiro, \& Tribess (2015), e que teve como um dos objetivos estimar fatores associados à dependência funcional em idosos, detectou o fato de que a incapacidade funcional para Atividades Básicas da Vida Diária associou-se a um aumento da idade, ao consumo de bebidas alcoólicas, sintomatologia depressiva e fragilidade. De outra parte, as Atividades Instrumentais da Vida Diária foram associadas ao avanço da idade, analfabetismo, arranjo familiar multigeracional, hospitalização e fragilidade fisica, social e mental.

\subsection{Interferência das doenças crônicas nas condições de vida}

Esta temática concebeu a percepção dos idosos sobre a interferência das doenças crônicas quanto ao seu aspecto individual, enquanto as falas ressaltam uma conjunção de problemas que vem ao encontro de suas condições de vida. 
Ao se categorizar as falas, surgem sentimentos aflitivos, como:

"Agonia no coração" (E1),"Dor nas pernas e não poder sair"(E2), "Diabetes causa dor nas pernas e gastura"(E5), "Hipertensão... não realizar minhas atividades"(E4), "A pressão dá muito problema”(E3), "Não está tudo ok, o dinheiro é curto”(E6), "Não poder fazer as atividades que fazia” (E7).

As comorbidades na pessoa idosa representam uma diminuição na mobilidade fisica, social e econômica que, para eles, causam contrangimento pela falta de liberdade para tomar decisões inerentes a sua vida. A independência do ser humano torna-se um fator essencial para a sobrevivência.

Pesquisadores, ao identificarem alta prevalência de dependência $(38,6 \%)$ entre idosos, sugerem que essa dependência receba influência das características sociodemográficas e de saúde expressas pelos referidos idosos. Enfatizam, portanto, ser fundamental a avaliação das capacidades e habilidades funcionais dos idosos, visando à prevenção de perda da independência e manutenção da autonomia, bem como à redução das internações hospitalares e dos gastos públicos (Sudré, Reiners, Nakagawa, Azevedo, Floriano, \& Morita, 2012).

\subsection{Doenças crônicas e o desempenho funcional}

No que concerne à perda da funcionalidade e ao dimensionamento dos sentimentos do idoso consigo mesmo ante seu desempenho funcional, afloram expressões relatando que:

"Não sinto alegria, tenho vontade de morrer"(E8), "Não me sinto bem... Era acostumada a fazer as tarefas de casa"(E9), "Não fazer as atividades domésticas que fazia antes..."(E10), "Falta de coragem" e "Me sinto cansado, Não posso fazer o que fazia"(E11, E7), "Não me sinto bem", "Gostaria de fazer minhas atividades, mas não consigo(E12), Recebo ajuda dos meus filhos"... Me sinto constrangida ..."(E13, E 6).

Corroborando estes achados, investigadores dessa matéria ressaltam que, relativamente a idosos com dificuldade para realizar as Atividades Básicas da Vida Diária e Atividades Instrumentais da Vida Diária, as chances de eles relatarem uma percepção negativa da saúde foram de 3,20 e 2,04 vezes maiores do que as daqueles sem estas dificuldades.

Os resultados evidenciam, também, que as pessoas impedidas de realizar, por algum motivo de saúde, quaisquer das atividades habituais, tendem a eleger a saúde como ruim, 
quando em comparação com os que não tiveram tal impasse (Zanesco, Bordin, Santos, Müller, \& Fadel, 2018).

Na lição de Freitas \& Soares (2019), o declínio funcional é uma indicação de cuidado, pois possui as principais dimensões de funcionalidade e afeta a autonomia e a independência da pessoa idosa. Alertam para o reconhecimento precoce, que permite a identificação de fatores desencadeantes das funções, provendo os profissionais de saúde com intervenções assistenciais baseadas nas especificidades do idoso. Além disso, estimula o debate para reformular políticas públicas e planos de intervenção, com o escopo de melhorar a autonomia e a independência daqueles postados nessas circunstâncias.

\subsection{Percepção da autonomia e a influência na dinâmica da vida}

Os depoimentos têm como referência a Autonomia e a Percepção do idoso sobre o ato de cuidar de si mesmo, sua interação com os outros e seu papel social. Eis o que emerge dos relatos:

\footnotetext{
"Sinto falta do passado, que podia trabalhar e hoje não consigo."(E5). "Triste, dependo dos outros".(E14). "Me sinto ruim, pois não posso fazer minhas tarefas." (E7). "Sinto-me triste por não realizar minhas atividades."(E1) "Me sinto mal, ruim, nada bom e sempre pedindo favor, triste. (E4). "Não resolver minhas coisas"...(E2). "Consigo conviver porque tenho ajuda“...Não consigo ler nem escrever“...(E10).
}

Os discursos ora reproduzidos representam a aflição, o incômodo, a dependência de outros que os idosos estão vivenciando. Evangelista, Bueno, Castro, Nascimento, Araújo \& Aires(2014) enfatizam sobre o sentimento de comoção relatado pelos seus entrevistados. Nas suas análises, a percepção do idoso parece não possuir causa específica comum, mas estar, sobretudo, relacionada com a história de vida no passado mais remoto. Para os autores, aceitar a realidade e os fatos como foram durante o ciclo de vida de cada um conforma uma tarefa difícil durante o envelhecimento. A realidade expressa demanda ações e políticas públicas, como suporte de profissionalização de cuidadores para a saúde e integração em redes de apoio social.

Nesta circunstância, a fragilidade de muitos idosos e a distância dos centros urbanos tornam sobejamente importantes as visitas domiciliares por parte dos profissionais da saúde que compõem a Estratégia Saúde da Família (ESF). Estas visitas permitem formar um mapa da 
realidade da comunidade, das famílias e de seus membros, bem como subsidiar estratégias e programas específicos de saúde (Magalhães, 2015).

\section{CONSIDERAÇÕES FINAIS}

Este estudo ressalta a subjetividade dos idosos em relação a dificuldades, conflitos e vulnerabilidade funcional, sentimentos aflitivos decorrentes das doenças crônicas. A perda da funcionalidade dimensiona sensações de dependencia e falta de liberdade, enquanto a autonomia reflete a percepção sobre a falta de cuidar de si mesmo, dinamismo, negligência na interação com os outros e seu papel social.

Sugere-se a necessidade de fortalecer a compreensão, conduta e acompanhamento próximo dos profissionais de saúde no melhor direcionamento sobre a funcionalidade e autonomia da população idosa.

As limitações deste escrito decorrem do fato de serem as experiências deste público um fenômeno complexo, bem assim por não haver o compartilhamento dos dados com os profissionais e cuidadores dos idosos. Mesmo assim, o trabalho, decerto, contribuirá com outro conhecimento qualitativo a mais, que poderá ser aplicado no cuidado à pessoa idosa pelos profissionais.

\section{REFERÊNCIAS}

Bardin, L.(2018). Análise de conteúdo. Lisboa: Edições 70, agosto.

Brasil. Ministério da Saúde. Portaria no 2528, de 19 de outubro de 2006. Aprova a Política Nacional de Saúde da Pessoa Idosa.Brasília: Ministério da Saúde; 2006.

Brasil. Portaria nº 2.436, de 21 de setembro de 2017. Ministério da Saúde. Secretaria de Atenção à Saúde. Departamento de Atenção Básica. Brasília: Ministério da Saúde.

Edwards, R., \& Holland, J. (2013). What is qualitative interviewing? Bloomsbury Academic. ISBN: HB: 978-17809-3852-3.

Evangelista, R. A., Bueno, A. A., Castro, P. A., Nascimento, J. N., Araújo, N. T., \&Aires, G. P. (2014). Percepções e vivências dos idosos residentes de uma instituição asilar. Revista da Escola de Enfermagem da USP; 48(Esp2):85-91.

Lawton MP, Brody EM. Assessment of older people: self-maintaining and instrumental activities ofdaily living. Gerontologist. 1969; 9(3):179-86.

Leandro-Franco, C; Murta, G. S. (2014). Prevenção e promoção da saúde mental no envelhecimento: conceitos e intervenções. Psicologia: ciência e profissão;34(2): 318-329.

Freitas, F. F. O., \& Soares, S. M. (2019). Clinical-functional vulnerability index and the dimensions of functionality in the elderly person. Revista da Rede de Enfermagem do Nordeste; 20:e39746. DOI: 10.15253/21756783.20192039746. 
Katz, S. (1983). Assessing self-maintenance: Activities of daily living, mobility, and instrumental activities of daily living. Journal American Geriatrics Society, 31, 721-727. https://doi. org/10.1111/j.1532-

5415.1983.tb03391.x

Magalhães, K. A. (2015). Envelhecimento e Cuidado: uma abordagem antropológica centrada na visão de agentes comunitários de saúde. (Tese Doutorado em Ciências da Saúde com concentração Saúde Coletiva). Belo Horizonte: Fundação Oswaldo Cruz, 2015. Disponível em: <https://www.arca.fiocruz.br/handle/icict/10757>. Acesso em: 15 jan. 2018.

Minayo, M. C. S. (2014). O desafio do conhecimento: pesquisa qualitativa em saúde. 14 edição, São Paulo: Hucitec, 2014.

Pereira, R. A., Souza, R. A. A.,\& Vale, J. S. (2015). O Processo de Transição Epidemiológica No Brasil: uma Revisão de Literatura. Revista Científica da Faculdade de Educação e Meio Ambiente, [S.I.], 6 (1): 99-108.

Saintrain, M. V. L., \& Vieira, L. J. E. S. (2008). Saúde bucal do idoso: abordagem interdisciplinar. Ciências \& Saúde Coletiva; 13(4):1127-32.

Sgarbieri, V. C., \& Pacheco, M. T. B. (2017). Healthy human aging: intrinsic and environmental factors.BrazilianJournalof Food Technology; 20: e2017007.

Sudré, M. R. S., Reiners, A. A. O., Nakagawa, J. T. T., Azevedo, R. C. S., Floriano, L. A., \&, Morita, L. H. M. (2012). Prevalence of dependency and associated risk factors in the elderly. ActaPaulista de Enfermagem;25(6):947-53.

Virtuoso-Júnior, J. S., Martins, C. A., Roza, L. B., Paulo, T. R. S., Ribeiro, M. C. L., \&Tribess, S. (2015). Prevalência de incapacidade funcional e fatores associados em idosos. Texto Contexto Enfermagem, Florianópolis; 24(2): 521-9.

WHO. World report on ageing and health. 2015. World Health Organization, 20 Avenue Appia, 1211 Geneva 27, Switzerland. Disponível em: https://apps.who.int/iris/bitstream/handle/10665/186463/9789240694811_eng.pdf;jsessionid=1D9FEECC6A 95A5A964690A07B7AFDCE6? sequence $=1$

Zanesco, C., Bordin, D., Santos, C. B., Müller, E. V., Fadel, C. B.(2018) Factors determining the negative perception of the health of Brazilian elderly people. RevistaBrasileira de Geriatria e Gerontologia; 21(3): 283-292. 